Article

\title{
Kernel-Density-Based Particle Defect Management for Semiconductor Manufacturing Facilities
}

\author{
Seung Hwan Park ${ }^{\dagger}$, Sehoon Kim ${ }^{\dagger}$ and Jun-Geol Baek * (1) \\ Department of Industrial Management Engineering, Korea University, Seoul 02841, Korea; \\ udongpang@korea.ac.kr (S.H.P.); stanley.kimm@gmail.com (S.K.) \\ * Correspondence: jungeol@korea.ac.kr; Tel.: +82-2-3290-3396 \\ + Those authors contributed equally to this work.
}

Received: 2 January 2018; Accepted: 28 January 2018; Published: 1 February 2018

\begin{abstract}
In a semiconductor manufacturing process, defect cause analysis is a challenging task because the process includes consecutive fabrication phases involving numerous facilities. Recently, in accordance with the shrinking chip pitches, fabrication (FAB) processes require advanced facilities and designs for manufacturing microcircuits. However, the sizes of the particle defects remain constant, in spite of the increasing modernization of the facilities. Consequently, this increases the particle defect ratio. Therefore, this study proposes a particle defect management method for the reduction of the defect ratio. The proposed method provides a kernel-density-based particle map that can overcome the limitations of the conventional method. The method consists of two phases. The first phase is the acquisition of cumulative coordinates of the defect locations on the wafer using the FAB database. Subsequently, this cumulative data is used to generate a particle defect map based on the estimation of kernel density; this map establishes the advanced monitoring statistics. In order to validate this method, we conduct an experiment for comparison with the previous industrial method.
\end{abstract}

Keywords: kernel density estimation; particle defect management; particle map; semiconductor manufacturing process

\section{Introduction}

The worldwide semiconductor market is valued at 333 billion dollars [1]. As the semiconductor industry accounts for $10-15 \%$ of the total exports of the Republic of Korea, it has a significant influence on the market economy. Owing to the recent proliferation of electronic devices such as mobile phones and tablet PCs (Personal Computers), market competition is becoming increasingly fierce [2]. In the future, as the demand for the internet of things (IoT) products is expected to increase, most manufacturers endeavor to increase the production through maintenance of facilities [3]. The semiconductor manufacturing process consists of numerous steps. As shown in Figure 1, in the front-end process, the ingot is cut to produce wafers and the circuit is designed. The wafers are subsequently undergone fabrication (FAB), which consists of the following eight processes: oxidation, lithography, etching, strip \& clean, ion-implantation, chemical vapor deposition, metal deposition, and chemical mechanical planarization. Oxidation refers to a process whereby a thin and uniform silicon oxide film is formed by the chemical reaction of oxygen or water vapor and the surface of the wafer at a high temperature. Lithography is a process whereby patterns on the wafer is formed by photoresist coating, exposure, and development processes. Etching refers to the process whereby unnecessary portions are selectively removed by using the reactive gas to form a circuit pattern. Strip \& clean refers to the process of removing particle contamination generated on the wafer amid other ongoing processes. Ion-implantation is the process whereby characteristics of the electronic device are 
generated by the implantation of impurities converted to a gas form in the circuit pattern. Chemical vapor deposition is the process whereby the water vapor formed in the particles are formed by the chemical reaction of gases to form an insulating film. Metal deposition interconnects each circuit formed on the surface of a wafer with aluminum and copper wire. Chemical mechanical planarization is a process whereby the oxide film and the metal thin film coated on the wafer are ground and flattened using chemical and physical processes. The subsequent step is the back-end process, in which the probe test, the assembly, and the package tests are conducted sequentially. Thereby, the final product is manufactured. These processes each have their own facilities, and each facility includes several chambers where a wafer must be placed for fabricating. The above-mentioned processes occur within those chambers.

Wafer fabrication for semiconductor devices such as microprocessors, memories, digital signal processors, and consumer electronics applications involves a complex and lengthy process with 30-40 reentrant loops, and the above-mentioned processes (11-8)), as shown in Figure 1 [4]. This study focuses on these eight processes and the inter-process metrology.

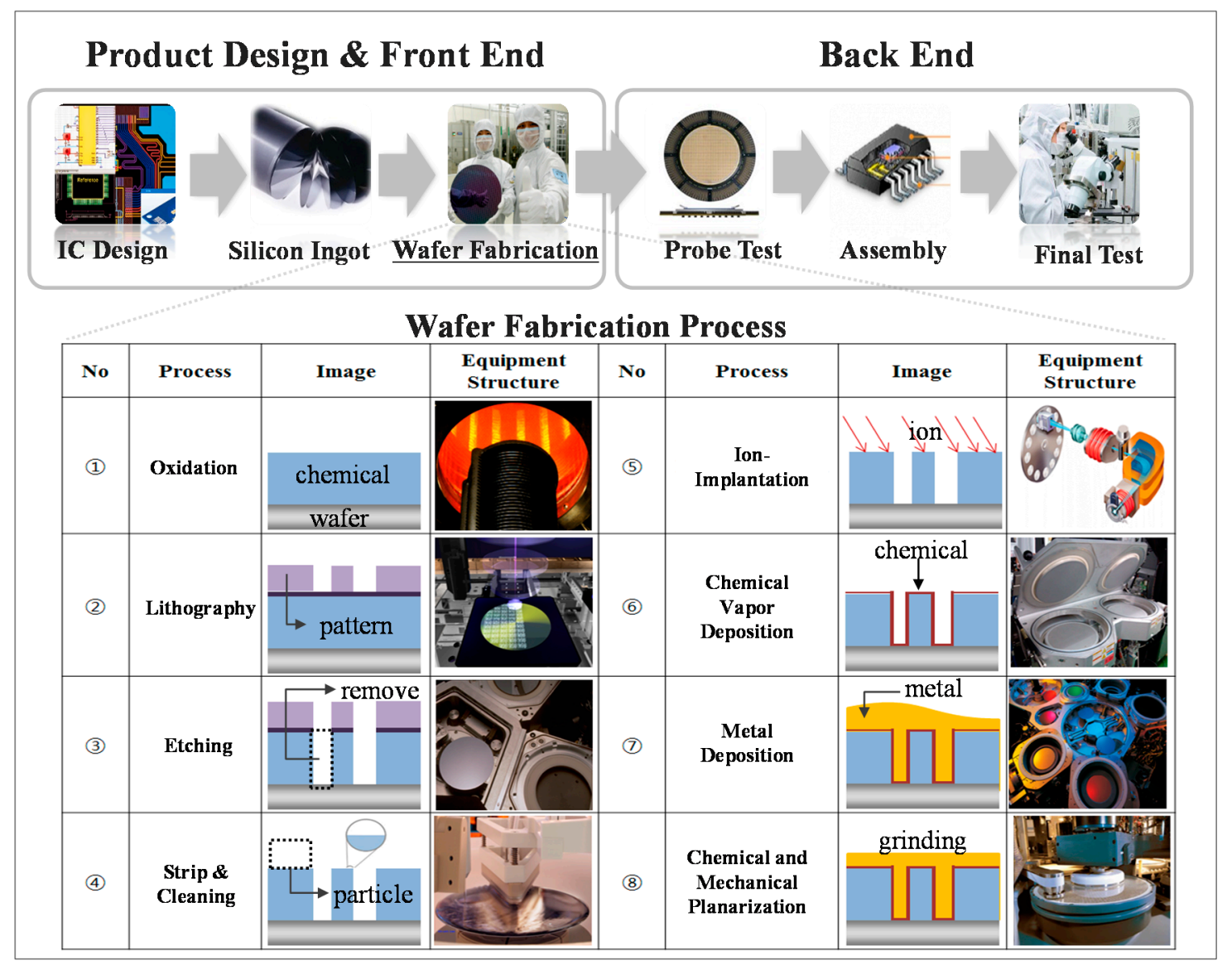

Figure 1. The semiconductor manufacturing process. IC, Integrated Circuit.

Semiconductor FAB includes various physical and chemical treatments and takes six to eight weeks. Therefore, there is a considerable difficulty in identifying causes of failure in the fabrication process, which involves numerous facilities. Figure 2 chronologically shows a pitch change in the lithography process used for fabricating DRAM (Dynamic Random-Access Memory), NAND (Negative-AND gate flash memory), and logic products that have been actively manufactured in recent years. The pitch on the $Y$-axis illustrates the integration capacity of the semiconductor products and significantly affects product yield, hence the increasing significance of defect management as current FAB processes design and fabricate sophisticated patterns [5]. 


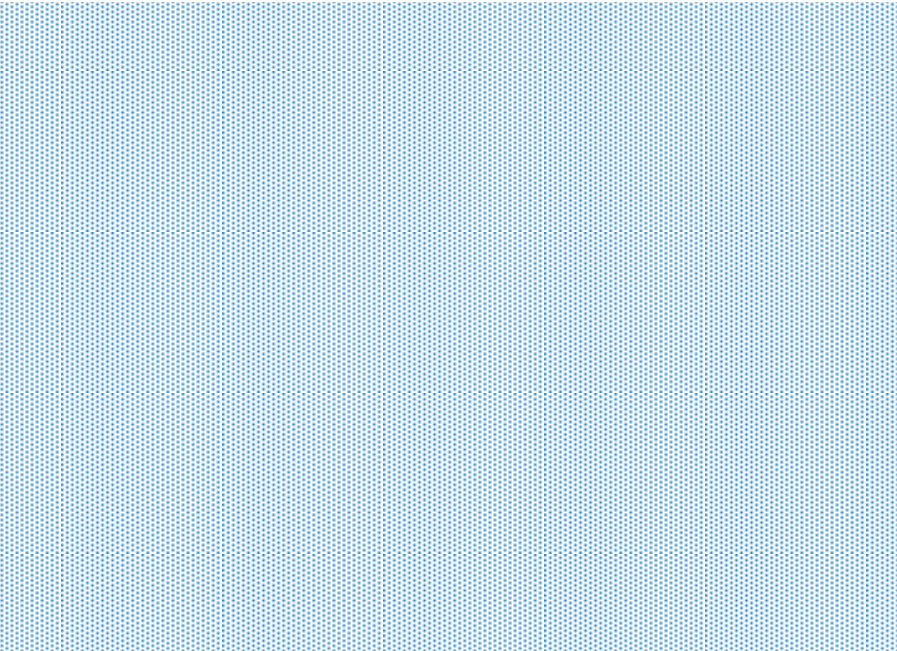

Figure 2. Pitch change in the lithography process (2002-2020). DRAM, Dynamic Random-Access Memory; NAND, Negative-AND gate flash memory.

The defects in a semiconductor manufacturing process are generally distributed as shown in Figure 3. The pie chart on the left shows that the particle defects account for $75 \%$ of the total defects. As shown in the pie chart on the right, $75 \%$ of the particle defects are caused by process equipment [6].
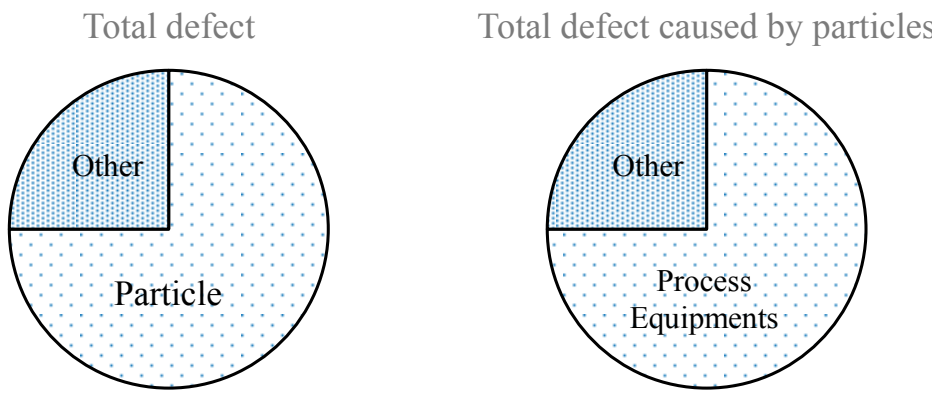

Figure 3. Distribution of defects in a semiconductor manufacturing process.

In the field of semiconductor manufacturing, a particle defect indicates a failure resulting from fine particles, such as dust, that are present on the wafers during FAB. The size of the particle defect owing to the manufacturing environment has remained unchanged, but the FAB process has been improved to develop capabilities for the fabrication of fine patterns. During one inspection, as shown in Figure 4, there was no defect when the pitch was $130 \mathrm{~nm}$, but a pitch of $45 \mathrm{~nm}$ resulted in a defect because the size of the particle was greater than the pitch size. Therefore, the structure and stability of the deposited chemical must be carefully controlled, and reduction in contamination in particular becomes increasingly crucial as device sizes shrink [7].

The particles in the chamber of a facility need to be monitored to maintain the chamber's stability. Current manufacturing processes manage particle defects through a simple control chart (c-chart) that monitors the number of particles. As these monitoring charts do not consider the distribution or density of the particles on the wafers, engineers do not know whether the particles are assignable or what the common causes are. Therefore, this study proposes a particle defect management method to overcome the limitation of this monitoring chart.

This paper is organized as follows. Section 2 reviews the conventional particle management method. Section 3 provides the details of the proposed method, and Section 4 presents the experimental results and evaluation. Finally, Section 5 concludes this study. 


\begin{tabular}{|c|c|c|}
\hline Pitch & Defect Image & Judgment \\
\hline $130 \mathrm{~nm}$ & & Non-defect \\
\hline $45 \mathrm{~nm}$ & & Defect \\
\hline
\end{tabular}

Figure 4. Change in the particle defect size with respect to the pitch.

\section{The Conventional Industrial Method}

In an actual semiconductor manufacturing process, conventional particle defect management involves manual action by engineers if, based on the monitoring chart, there is a cause for alarm. The procedure consists of three phases for the detection of the cause of the defect. In the first phase, when an alarm occurs, the insides of the manufacturing equipment are repeatedly cleaned. In the second phase, if the problem persists, the field engineers replace suspicious parts. In the final phase, if the previous replacement does not solve the prevailing problem, predictive maintenance (PM) of the equipment is performed. The second phase, in particular, consumes a substantial amount of time in terms of both decision-making and detection of the root cause, owing to an experience-based decision of the replacement. Therefore, reducing false alarms in the first phase is critical.

Among the aforementioned particle defect management phases, we focus on the first phase, wherein the particle defects are monitored. Particle defects in process equipment are primarily due to equipment aging. Other defects occur due to environmental factors. The counts control chart (c-chart) is commonly used to monitor particle defects. The c-chart is based on the assumption that the distribution of the number of nonconformities is sufficiently well fitted by a Poisson distribution [8]. However, in the actual semiconductor manufacturing process, the particle count does not follow a Poisson distribution [9]. Especially, in our case, random variables are not independent since the particles are cumulated with a time-varying property. Figure 5 shows the metrology equipment gauging the number of particles on the wafer. The number of particles, $N$, at each coordinate on the wafer is stored.

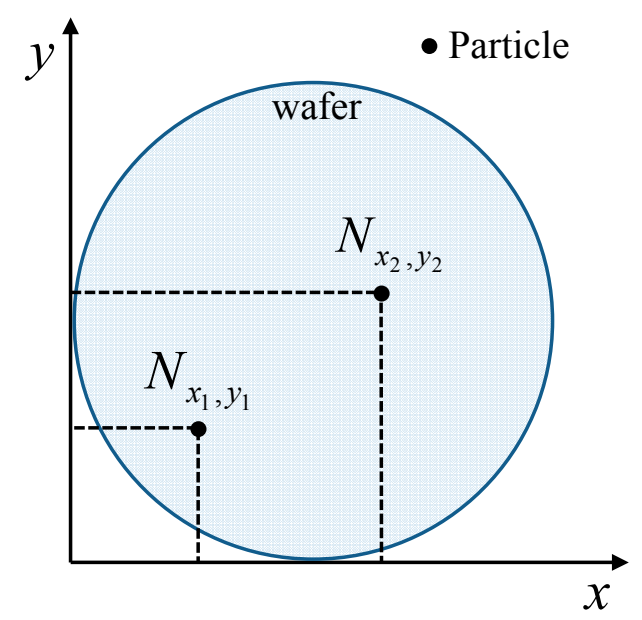

Figure 5. Particle measurements on a test wafer. 
Moreover, these particles indicate the status of the equipment, not of the wafer. Therefore, in conventional monitoring statistics, the particle defect (PD) that indicates the number of particles on a test wafer can be denoted as follows:

$$
P D=\sum_{i} N_{x_{i}, y_{i}}
$$

where $x_{i}$ and $y_{i}$ are the $i$-th particle coordinates, and $N_{x_{i}, y_{i}}$ indicates the number of particles at the designated coordinate.

The particle coordinates can be used to generate a wafer map to obtain the distribution and number of particles. Figure 6 depicts four illustrative particle defect maps for four processes. The particle defect maps indicate high-density particles at a particular location on the wafer and the location is correlated with the structure of the process equipment. As indicated by the dotted ellipse in each particle defect map, a high-density area indicates that an ongoing wafer has a potential defect. The particle defect map of the oxidation is also affected by the structure of the equipment. Field engineers have discovered a problem at the center position, where the gas is injected into the furnace, using the particle map. The lithography process shows a higher density at the edges of the wafer. The lithography process includes a photo resist (PR) coating using a high-speed rotation of the spinner equipment in the wafer process. Therefore, the remnants of the chamber affect the density of the wafer edges owing to the characteristics of the high-speed spinning process. Both the etching and strip \& clean processes exhibit the highest densities where the dotted ellipse is drawn. Engineers have checked the equipment that analyzes the causes of this phenomenon and discovered a particle resulting from the improper fastening of the O-ring in the chamber. Further, as the strip \& clean process includes multiple baths that form bias chemicals at the bottom wafer, the particle defect map indicates high densities of the particles in the bottom area.

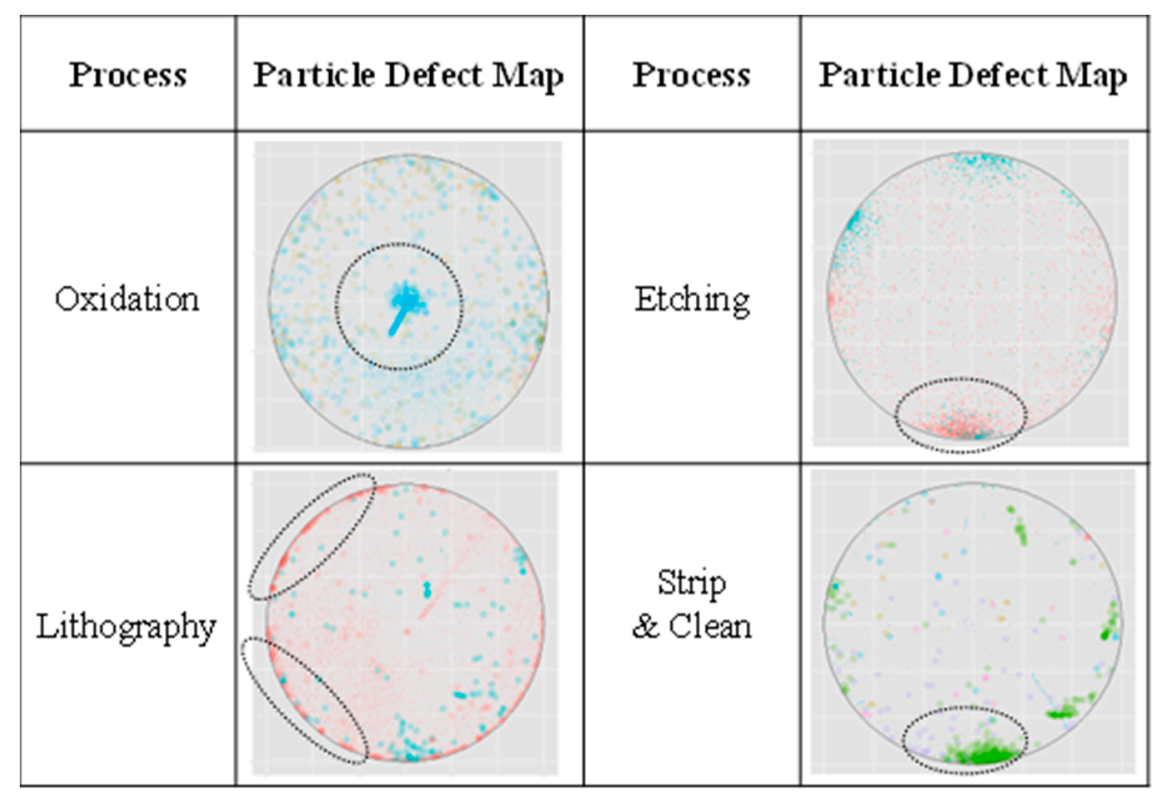

Figure 6. Particle maps of the four processes.

A real domain conducts monitoring based on the control chart in Equations (2)-(4) to monitor the particle counts [9].

$$
\begin{gathered}
\text { Center line }=\overline{P D} \\
\text { Upper control limit }=\overline{P D}+C V \times \sqrt{\overline{P D}} \\
\text { Lower control limit }=\overline{P D}-C V \times \sqrt{\overline{P D}} .
\end{gathered}
$$


However, $C V$ (critical value) is decided through the experience of engineers because the particle defect cannot assume a specific distribution. Moreover, since the particle count is always positive, Lower control limit is zero. Therefore, the actual process determines the abnormalities in the facility using the threshold of the particle count, as shown in Figure 7.

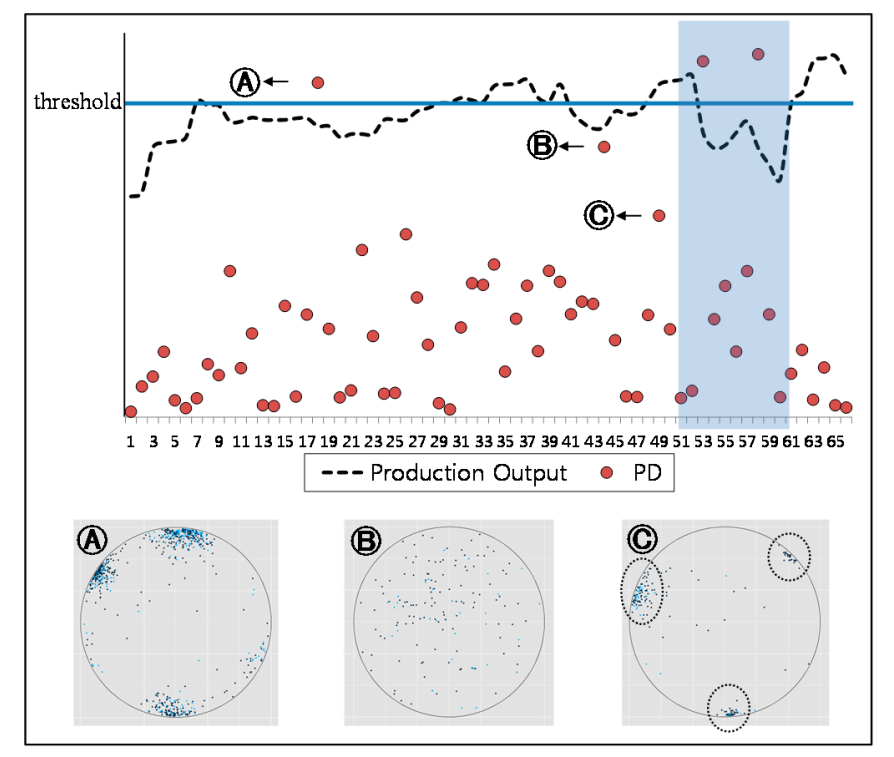

Figure 7. Illustration of the conventional monitoring method. PD, particle defect.

Figure 7 describes both the monitoring chart and the particle maps of the wafers required for observing the particles with specific equipment. In the monitoring chart, the horizontal axis is the wafer index and the vertical axis is the monitoring statistic, $P D$. The dashed-polygonal line indicates production yield and the solid straight line indicates the criterion that distinguishes between a non-defect and a defect. As shown in the chart, Wafer A is beyond the threshold, whereas Wafers B and $C$ are within the threshold. Although both Wafers $B$ and $C$ are within the threshold, they have different distributions, as shown by their respective maps. The three wafer maps depict their respective particle distributions. Wafer A shows high-density particles because $P D$ is beyond the threshold. The particles in Wafers $B$ and $C$ are differently distributed, whereas the monitoring chart indicates that $P D$ is within the threshold for both wafers. The particles on Wafer $\mathrm{B}$ are uniformly distributed, and the particles on Wafer $C$ are concentrated around the edges of the wafer. Consequently, despite a smaller $P D$, the particles concentrated on Wafer $C$ can induce fatal risks, including out-of-threshold wafers or yield reductions, as shown by the shaded rectangular area of the plot. Therefore, since it is important to identify particle distributions, as in Wafer C, this study proposes a new monitoring method considering dense particles.

\section{The Proposed Method}

The proposed method consists of two stages. The first stage consists of the particle map based on the estimation of kernel density and the cumulative particle data. The second stage proposes a new monitoring statistic, calculated from the probability of the kernel function based on the particle map.

\subsection{Particle Map Generation Based on the Kernel Density}

As the particle map indicates only the distribution of the particles using the cumulative data, the density of the particles should be considered. Therefore, this method utilizes a function for the estimation of kernel density. Kernel density estimation is a non-parametric method for estimating the probability density of a dataset. As the particle map consists of two dimensions, the $X$-axis and $Y$-axis of the wafer, this map is constructed using multivariate kernel density estimation. Considering 
$\left\{x_{1}, y_{1}\right\},\left\{x_{2}, y_{2}\right\}, \ldots,\left\{x_{n}, y_{n}\right\}$ as the sample data of two-dimensional vectors and $n$ as the number of particles, the kernel density estimation function is expressed as [10], where $H$ is the scale coefficient of the kernel function and $K$ is the Gaussian kernel function, which is a symmetric multivariate density.

$$
\hat{f}_{H}(x, y)=\frac{1}{n} \sum_{i=1}^{n} K_{H}\left\{(\bar{x}, \bar{y})-\left(x_{i}, y_{i}\right)\right\}
$$

The scale coefficient $H$ of Gaussian kernel function $K$ is estimated using a well-supported rule [11] and is dependent on the performance of particle inspection equipment. $H$ means the estimated sigma. In this study, we selected 99,370 as the value of $H$. However, this value can vary depending on the resolution of image data from equipment. Consequently, this function calculates the density $\hat{f}_{H}(x, y)$ of the wafer. We build a three-dimensional kernel-density-based particle map using $\hat{f}_{H}(x, y)$ in the $X$-axis and $Y$-axis. Figure $8 \mathrm{a}, \mathrm{b}$ illustrate the particle map and the kernel-density-based particle map, respectively. In Figure $8 \mathrm{a}$, the dotted area indicates dense particles, and Figure $8 \mathrm{~b}$ shows a high density at the corresponding area in Figure $8 \mathrm{a}$. Hence, Figure $8 \mathrm{~b}$ shows the probability density of the corresponding particles of the map in Figure 8a. This particle map contains dense particles regardless of the total count of the particles.



(a)

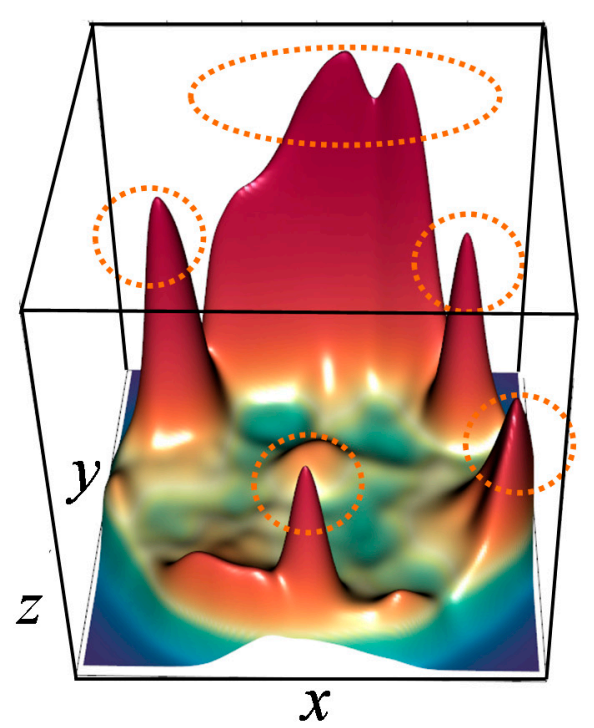

(b)

Figure 8. The conventional and new particle maps: (a) particle map; (b) kernel-density-based particle map.

As these dense particles can cause an abnormal state in the facility, this study devises new monitoring statistics that consider the density of the particles using the kernel-density-based particle map.

\subsection{New Monitoring Statistics Using the Proposed Map}

As mentioned in the previous section, the conventional method uses $P D$ for monitoring. This chapter describes new monitoring statistics that can replace the conventional statistics. The kernel-density-based particle map shows the probabilities at all the points on the wafer, as shown in Figure $8 \mathrm{~b}$. While the original particle map shows only the location of the particles, 
the kernel-density-based particle map includes the density of the particles at a location. In the case of a kernel-density-based particle defect, the expected value $E$ of the particle defect is calculated as

$$
E=\frac{\hat{f}_{H}(x, y)}{\sum_{i=1}^{n} \hat{f}_{H}\left(x_{i}, y_{i}\right)} \times n
$$

where $n$ is the total count of the particles, $\hat{f}_{H}(x, y)$ denotes the density of the particle map, and $\frac{\hat{f}_{H}(\mathrm{x})}{\sum_{i=1}^{n} \hat{f}_{H}\left(\mathrm{x}_{i}\right)}$ is the probability based on the density. The expected value indicates a new particle defect at the location of the particle. Therefore, according to the new monitoring statistics, the kernel-density-based particle defect $(K D P D)$ is calculated as

$$
K D P D=P D+\sum_{i} E_{x_{i}, y_{i}}
$$

where $x_{i}$ and $y_{i}$ are $i$-th particle coordinates and $E_{x_{i}, y_{i}}$ indicates the expected value of each particle at the designated coordinates.

The sum of $E_{x_{i}, y_{i}}$ indicates the number of particles considering the densities of the wafer and defines the new monitoring statistics. Thus, the monitoring chart utilizes KDPD to detect potential particle defects. Figure 9 depicts the comparisons between the results of certain $P D$ and KDPD. While Wafers A, B, and C exhibit identical PDs, KDPD assumes different values for each wafer. As KDPD is derived using the particle distributions on the map in Figure 9, it indicates a density-based $P D$. The dense particles, shown at the bottom of the Wafer $C$ map, influence monitoring performance.

\begin{tabular}{|c|c|c|c|}
\hline Wafer & A & B & $\mathbf{C}$ \\
\hline $\mathrm{PD}$ & 50 & 50 & 50 \\
\hline KDPD & 54.5 & 55.5 & 71 \\
\hline $\begin{array}{c}\text { Particle } \\
\text { Defect } \\
\text { Image }\end{array}$ & & & \\
\hline
\end{tabular}
Thus, KDPD dynamically changes in accordance with the particle distribution.

Figure 9. Comparative particle defect (PD) and kernel-density-based particle defect (KDPD).

\section{Experimental Results}

This section describes an experiment for verifying the proposed method and its results. The particle maps used for this experiment were retrieved from the etching process. As several "killer-particles" originate from the deposition of a layer of by-product on the inside of the plasma-etching chamber, the etching process is sensitive to particles [12]. Therefore, we used particle data from a real etching process in a Korean semiconductor manufacturing company, obtained over a period of five months. The entire dataset consists of 600 wafers, generating 600 particle maps. Among these particle maps, we defined the particle defect types using field knowledge. A dense particle indicates that the facility is abnormal. Therefore, the dataset with dense and even particles was selected. The four defect types are shown in Figure 10. Our dataset consists of 300 normal wafers, 100 wafers of Type 1, 100 wafers of Type 2, 50 wafers of Type 3, and 50 wafers of Type 4. These types were determined by experienced engineers.

As shown in Figure 10, Type 1 shows an illustrative particle map of a small defect. This map includes only one particle cluster, shown on the left side of the map. Type 2 map has two or more 
small defects. The Type 3 map has only one large-sized defect and the Type 4 map shows the particle distribution on the entire surface of the wafer.

According to the specified defect types, we generated four datasets for the experiment, as shown in Table 1.

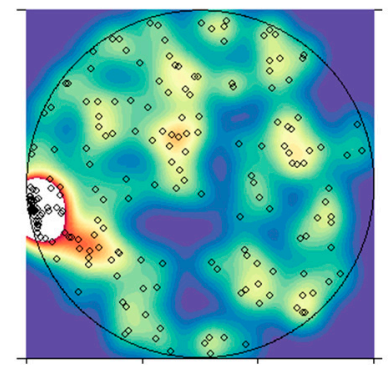

Type 1: small defect



Type 3: large defect

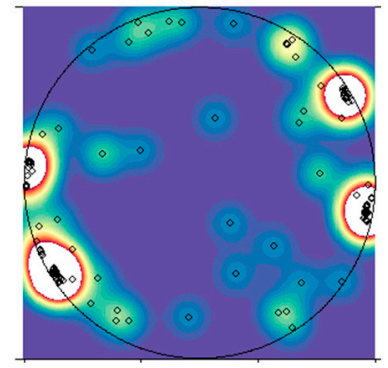

Type 2: medium defect



Type 4: huge defect

Figure 10. Levels of the defects in the etching process.

Table 1. Defect datasets.

\begin{tabular}{cc}
\hline Dataset & Combination of Defect Types \\
\hline Dataset 1 & Types $1,2,3$ and 4 \\
Dataset 2 & Types 2,3 and 4 \\
Dataset 3 & Types 3 and 4 \\
Dataset 4 & Type 4 \\
\hline
\end{tabular}

Dataset 1 includes non-defect wafer images and wafer images with Defect Types 1, 2, 3 and 4 . On the other hand, Dataset 4 consists of non-defect wafers and Type 4 defect wafers only. In order to validate the monitoring performance for these datasets, we evaluated the use of $K D P D$, compared to the use of the conventional monitoring statistics, to identify a defect.

We used the monitoring method illustrated in Figure 7 to classify non-defect and defect particle maps. The threshold in the monitoring chart corresponds to the upper control limit (UCL) in the statistical process control chart. Therefore, we compared the classification performance of the particle defect map by adjusting the decision threshold of the defect/non-defect. Further, we used the receiver operating characteristic (ROC) curve, a graphical plot that illustrates the performance of a binary classification. Moreover, in order to compare these curves, the area under the ROC (AUROC) curve was considered [13]. A broader AUROC can detect a particle defect more accurately. As defect classification is more important than non-defect classification, the ROC curve uses a false negative rate for the $X$-axis and a true negative rate for the $Y$-axis. Figure 11 depicts the ROC curves for both PD and KDPD of the four datasets defined in Table 1 , and the AUROC examines the monitoring performances.

The ROC curves of Datasets 1, 2 and 3 show that the AUROCs of KDPD are broader than those of PD. Therefore, KDPD outperforms PD. Moreover, the results indicate an increase in the performance gap between PD and KDPD in proportion to the degree of the defects. On the other hand, Dataset 4 demonstrates that the conventional statistics (PD) outperforms KDPD. This dataset includes the Type 
4 defect as specified in Table 1 and is referred to as a fatal defect. This can be monitored using either PD or KDPD. Therefore, the proposed KDPD is appropriate for detecting Type 1, 2, and 3 defects. Since the Type 1, 2 and 3 defects indicate potential factors that can result in fatal defectives of Type 4 , field engineers can detect critical defects in early stages. Table 2 represents the area under the ROC curves depicted in Figure 11. In Datasets 1, 2 and 3, the area of KDPD is much larger than PD. However, in the case of Dataset 4, the area of KDPD is slightly smaller than PD.

Dataset 1

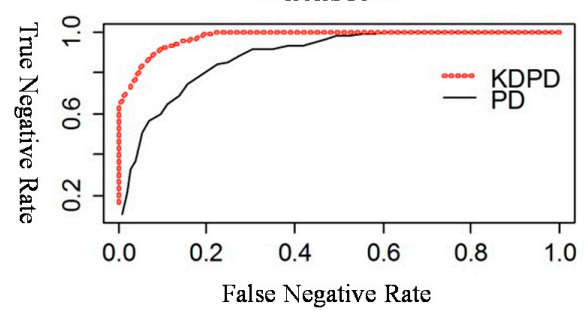

Dataset 3

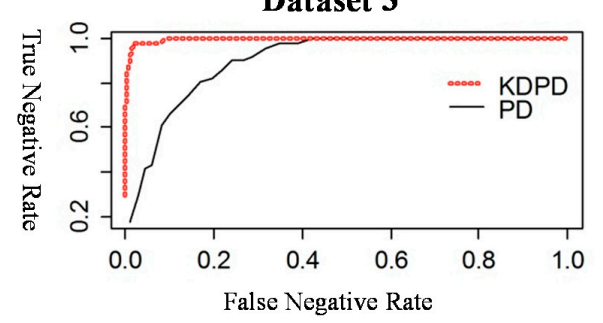

Dataset 2



Dataset 4



Figure 11. Receiver operating characteristic (ROC) curves of the four datasets including each fault type.

Table 2. Area under the ROC (AUROC) (If AUROC $=1$, then the test is perfect. If $A U R O C=0.5$, then the test is worthless) ${ }^{1}$.

\begin{tabular}{ccc}
\hline Dataset & PD & KDPD \\
\hline Dataset 1 & 0.79 & 0.92 \\
Dataset 2 & 0.81 & 0.93 \\
Dataset 3 & 0.84 & 0.98 \\
Dataset 4 & 0.99 & 0.97 \\
\hline
\end{tabular}

${ }^{1}$ ROC; Receiver operating characteristic; PD, particle defect; KDPD, kernel-density-based particle defect.

\section{Conclusions}

This study is significant because the proposed method utilizes the real data of the particles in the chamber of a semiconductor process. Novel monitoring statistics that efficiently identify the particles on the wafers in a chamber are proposed in this study. These new statistics are based on multivariate kernel density estimation and indicate the densities of the particles. Thorough management is essential for reducing the failures caused by particles in a semiconductor manufacturing process. This study presents a particle defect management method using a kernel-density-based particle map for improving the conventional method of monitoring the number of particles. In particular, the main contribution of this study is the development of new monitoring statistics. The new statistics that consider the distribution of particles, when applied to an actual process, produce the following three effects. Firstly, the proposed method can reduce the number of unnecessary replacements. Secondly, according to a field internal review, when this study is applied to an actual process, a reduction of approximately $30 \%$ in the meantime to repair (MTTR), i.e., the time to find the cause of a failure, is expected. Third, regardless of the number of particles, dense particles increase the possibility of further failures. Therefore, using the proposed particle maps, it is possible to detect defects and predict future failures. 
Since the semiconductor manufacturing processes are becoming finer, particle defect management becomes increasingly important. If the proposed kernel-density-based particle map can be applied to an actual process, it is expected to improve both the yield and the quality of the semiconductor product. The size of particle defect has a large influence on yield. The current wafer size is $300 \mathrm{~mm}$, but the wafer size will grow and the defect rate will increase as the process is refined. Therefore, in the future, it is necessary to identify the relationship between the particle map and both the yield and quality of the wafer.

Acknowledgments: This work was supported by the National Research Foundation of Korea (NRF) grant funded by the Korea government (MSIP) (NRF-2016R1A2B4013678). This work was also supported by the BK21 Plus (Big Data in Manufacturing and Logistics Systems, Korea University) and by the Samsung Electronics Co., Ltd.

Author Contributions: Seung Hwan Park and Sehoon Kim contributed equally to this work as co-first authors. They designed and implemented the algorithm to solve the defined industrial problem. Jun-Geol Baek validated the proposed algorithm and guided the research. All authors read and approved the final manuscript.

Conflicts of Interest: The authors declare no conflict of interest.

\section{References}

1. Gartner. Available online: https://www.gartner.com/newsroom/id/3282417 (accessed on 27 December 2017).

2. Chik, M.A.; Saidin, M.H.; Hashim, U. Industrial Engineering Roles in Semiconductor Fabrication. In Proceedings of the 11th Asia Pacific Industrial Engineering \& Management Systems Conference, Melaka, Malaysia, 7-10 December 2010.

3. Solid State Technology. Available online: http://electroiq.com/blog/2015/09/the-future-of-mems-in-theiot (accessed on 27 December 2017).

4. Chien, C.F.; Wang, W.C.; Cheng, J.C. Data mining for yield enhancement in semiconductor manufacturing and an empirical study. Expert Syst. Appl. 2007, 33, 192-198. [CrossRef]

5. Meiling, H. EUVL-Getting ready for volume introduction. In Proceedings of the SEMICON West 2010, San Francisco, CA, USA, 12-16 July 2010.

6. Ahn, K.-H.; Miller, S.J. Particle sampling and element analysis from semiconductor manufacturing equipment. In Proceedings of the International Symposium on Cleanroom Technology and Contamination Control, Seoul, Korea, 19-20 September 1990.

7. Aziz, F.A.; Ahmad, I.H.; Zulkifli, N.; Yusuff, R.M. Particle Reduction at Metal Deposition Process in Wafer Fabrication. In Manufacturing System; Aziz, F.A., Ed.; InTech: Rijeka, Croatia, 2012; pp. 1-26, ISBN 978-953-51-0530-5.

8. Montgomery, D.C. Introduction to Statistical Quality Control, 7th ed.; Wiley: New York, NY, USA, 2013; pp. 317-335, ISBN 978-1118146811.

9. Kawamura, H.; Nishina, K.; Higashide, M. Control Charts for Particles in the Semiconductor Manufacturing Process. Econ. Qual. Control 2008, 23, 95-107. [CrossRef]

10. Silverman, B.W. Density Estimation for Statistics and Data Analysis; Chapman \& Hall: London, UK, 1986; pp. 7-11, ISBN 0-412-24620-1.

11. Venables, W.N.; Ripley, B.D. Modern Applied Statistics with S-PLUS, 3rd ed.; Springer: New York, NY, USA, 1999; pp. 113-148, ISBN 978-1-4757-3123-1.

12. Lee, H.-J.; Lin, S.-Y.; Lin, I.-T.; Wei, K.-L.; Chang, S.-Y.; Lian, N.-T.; Yang, T.; Chen, K.-C.; Lu, C.-Y. Post Etch Killer Defect Characterization and Reduction in a Self-aligned Double Patterning Technology. In Proceedings of the 2011 IEEE/SEMI Advanced Semiconductor Manufacturing Conference, Saratoga Springs, NY, USA, 16-18 May 2011.

13. Kang, S.; Cho, S.; An, D.; Rim, J. Using Wafer Map Features to Better Predict Die-Level Failures in Final Test. IEEE Trans. Semicond. Manuf. 2015, 28, 431-437. [CrossRef]

( 2018 by the authors. Licensee MDPI, Basel, Switzerland. This article is an open access article distributed under the terms and conditions of the Creative Commons Attribution (CC BY) license (http://creativecommons.org/licenses/by/4.0/). 